\title{
Influência da atividade e inatividade física na composição corporal e adiposidade central
}

\author{
Rômulo Araújo Fernandes ${ }^{1}$ \\ Juliano Casonatto ${ }^{2}$ \\ Diego Giuliano Destro Christofaro ${ }^{2}$ \\ Camila Buonani ${ }^{3}$ \\ Arli Ramos de Oliveira ${ }^{2}$ \\ Ismael Forte Freitas Júnior ${ }^{3}$
}

${ }^{1}$ Instituto de Biociências, UNESP - Univ Estadual Paulista, Campus de Rio Claro, Departamento de Educação Física, SP, Brasil

${ }^{2}$ Centro de Educação Física e Esporte, UEL, Londrina, PR, Brasil

${ }^{3}$ Faculdade de Ciências e Tecnologia, UNESP - Univ Estadual Paulista, Campus Presidente Prudente, Departamento de Educação Física, SP, Brasil

\begin{abstract}
Resumo: Analisar a influência da atividade física e comportamentos sedentários em indicadores de gordura corporal total e central em adolescentes do sexo masculino. Estudo transversal com 60 estudantes do sexo masculino com idades variando entre 11 e 14 anos. Foram avaliados a massa corporal, estatura, dobra cutânea tricipital, circunferência de cintura, percentual de gordura corporal (impedância bioelétrica) e o nível de atividade física por meio de questionário. A prevalência de inatividade física foi de $35 \%$, o excesso de gordura corporal total e central foram observados em $38,3 \%$ e $48,3 \%$ da amostra, respectivamente. Houve associação dos comportamentos sedentários com a gordura corporal total e central ( $R C=5.2$ e $R C=6.4$, respectivamente). Não houve associação dos mesmos indicadores com a atividade física. Os resultados indicaram que a adoção de comportamentos sedentários está associada ao desenvolvimento da obesidade total e central entre adolescentes do sexo masculino.
\end{abstract}

Palavras-chave: Adolescentes, Obesidade, Atividade física, Inatividade física.

\section{Physical activity and inactivity effect on body composition and central adiposity}

\begin{abstract}
To analyze influences of the physical activity and sedentary behaviors on indicators of both central and total body fat in male adolescents. Cross-sectional study with 60 male students of age range from 11 to 14 years old. It were evaluated the body mass, height, triceps skinfold, waist circumference, body fat percentage (bioelectrical impedance) and the physical activity level through questionnaire. The physical inactivity prevalence was of $35 \%$, and the excessive total and central body fat were observed in $38.3 \%$ and $48.3 \%$ of the sample, respectively. There was association of the sedentary behaviors with the excessive total and central body fat $(\mathrm{OR}=5.2$ e OR=6.4, respectively), however there was not for physical activity. The adoption of sedentary behaviors is associated at the development of the total and central obesity among male adolescents.
\end{abstract}

Key Words: Adolescents, obesity, physical activity, physical inactivity.

\section{Introdução}

Estudos indicam que, na população jovem brasileira, a ocorrência de sobrepeso e obesidade triplicou nas últimas décadas e constitui um importante problema de saúde pública; uma vez que paralelamente ao esse crescimento, observase também um expressivo aumento na ocorrência de outros fatores de risco ao desenvolvimento de doenças crônicas (WANG, MONTEIRO, POPKIN, 2002; FERREIRA, OLIVEIRA, FRANCA, 2007).

Nessa perspectiva, estudos têm indicado que o comportamento das variáveis de natureza comportamental, como a prática habitual de atividades físicas [PHAF] (inatividade física) e a ingestão alimentar (inadequada ingestão alimentar), estão diretamente associadas ao desenvolvimento do sobrepeso/obesidade (BOUCHARD, 2000).

Além disso, recentes estudos têm indicado que jovens insuficientemente ativos apresentam chances elevadas de desenvolver um acúmulo excessivo de gordura corporal, e que a inatividade física entre populações adultas está relacionada ao desenvolvimento de inúmeras patologias (RIBEIRO et al., 2006; CHENOWETH e LEUTZINGER, 2006). 
A maior PHAF relaciona-se de forma negativa com a gordura corporal (GUTIN et al., 2005). Entretanto, pesquisas mais recentes têm apontado que a PHAF de maior intensidade e hábitos sedentários são variáveis que influenciam, de forma independente os indicadores de gordura corporal e o risco cardiovascular (EKELUND et al., 2006; FERNANDES et al., 2006; FERNANDES et al., 2008).

Em virtude de se saber que a distribuição de gordura também com aumento do risco de desenvolver doenças crônicas e do conhecimento escasso da relação desse aumento com a PHAF de jovens brasileiros, decidiu-se realizar 0 presente estudo cujo objetivo foi analisar a existência de possíveis associações entre a PHAF e comportamentos sedentários com o desenvolvimento da obesidade e gordura corporal central em jovens do sexo masculino.

\section{Métodos}

\section{Amostra}

Para a composição da amostra, foram realizados anúncios na imprensa, convidando jovens de ambos os sexos com idade entre sete e 14 anos que acreditassem apresentar o estado de sobrepeso/obesidade e que desejassem participar de um programa de exercícios físicos e reeducação alimentar desenvolvido pela Universidade Estadual Paulista (UNESP Presidente Prudente). De todos os inscritos, foram selecionados apenas aqueles do sexo masculino, com idade entre 11 e 14 anos, perfazendo um total de 60 indivíduos.

Todos os jovens envolvidos na pesquisa e seus respectivos responsáveis foram informados quanto aos objetivos do estudo, receberam esclarecimentos quanto aos métodos utilizados na pesquisa e garantiu-se aos mesmos o direito de desistir do experimento a qualquer momento sem sofrer qualquer tipo de ônus. Participaram da amostra apenas os jovens que apresentaram 0 "Termo de Consentimento Livre e Esclarecido" devidamente assinado por seus responsáveis e os métodos utilizados no presente estudo foram aprovados pelo Comitê de Ética em Pesquisa local (Processo no. 087/2006).

\section{Prática Habitual de Atividades Físicas e Comportamentos Sedentários}

$\mathrm{Na}$ presente investigação, as informações de PHAF e exposição à comportamentos sedentários foram coletados por meio da aplicação de questionário. O instrumento de medida adotado foi o Questionário Internacional de Atividade Física (IPAQ), em sua versão curta. Para que toda a amostra do estudo preenchesse o instrumento sob condições similares, os sujeitos envolvidos no estudo, foram instruídos por um avaliador sobre a forma correta de preenchimento e tiveram suas dúvidas esclarecidas. Em sala de aula, os questionários foram preenchidos e devolvidos no mesmo dia. Durante o preenchimento não foi permitida a comunicação entre os avaliados e três avaliadores ficaram à disposição para esclarecer qualquer dúvida durante o processo de preenchimento. De posse dos dados fornecidos pelo IPAQ, foi calculado o tempo diário, em minutos ( $\mathrm{min} / \mathrm{dia}$ ), despendido por cada indivíduo nas diferentes intensidades de esforço: alta, moderada, leve e as atividades de repouso. Com base no somatório do tempo gasto em atividades intensas e moderadas, e por meio das recomendações mínimas de prática habitual de atividades físicas para crianças e adolescentes $(60 \mathrm{~min} / \mathrm{dia})$ (BIDDLE, CAVILL, $\underline{\text { SALLIS, }}$ 1998) os jovens foram classificados como ativos ( $\geq 60 \mathrm{~min} / \mathrm{dia})$ ou inativos $(<60 \mathrm{~min} / \mathrm{dia})$. O tempo em atividades de repouso foi utilizado com indicador de exposição a comportamentos sedentários, onde os 30 maiores tempos individuais foram classificados como sendo "expostos" e os 30 menores como "não expostos" a comportamentos sedentários.

\section{Antropometria, Gordura Corporal Total e Central}

As medidas antropométricas referentes à variável massa corporal, estatura e dobra cutânea tricipital (DTR), circunferência da cintura (CC) e impedância bioelétrica (BIA) foram coletadas por professores e alunos do curso de Educação Física da UNESP, todos avaliadores previamente treinados para a coleta das mesmas.

A massa corporal da amostra foi aferida por meio de uma balança da marca Filizola com precisão de $0,1 \mathrm{~kg}$ e capacidade máxima de $150 \mathrm{~kg}$. Medidas de estatura foram obtidas por meio da utilização de um estadiômetro fixo de madeira com extensão de dois metros e precisão de $0,1 \mathrm{~cm}$. A DTR foi aferida com a utilização de um adipômetro da marca Lange (Cambridge Scientific Industries) com valores expressos em milímetros $(\mathrm{mm})$. As medidas foram realizadas no hemicorpo direito do indivíduo em triplicata, onde adotou-se o valor mediano. Todas as variáveis antropométricas foram coletadas seguindo as recomendações apresentadas na literatura por 
Lohman, Roche e Martorell (1982). Além disso, baseando-se nos valores de referência (MUST, DALLAL, DIETZ, 1991) para DTR, o percentil 85 $(\geq$ P85) de cada idade foi considerado como indicadores sobrepeso/obesidade.

A circunferência de cintura (CC) foi adotada no presente estudo como indicador de obesidade abdominal, sendo as medidas tomadas em duplicata na mínima circunferência entre a crista ilíaca e a última costela, com a utilização de uma fita metálica com precisão em milímetros $(\mathrm{mm})$ (LOHMAN, ROCHE, MARTOREL, 1988). Além disso, baseando-se em estudos recentes que comprovam sua eficiência na indicação dos componentes da SM, os valores críticos para CC específicos para sexo e idade foram adotados para indicar a presença da obesidade abdominal (ALMEIDA et al., 2007; TAYLOR et al., 2000).

A resistência e a reatância corporal (ohm- $\Omega$ ) foram aferidas com a utilização de um analisador portátil de composição corporal (BIA Analyzer 101Q, RJL Systems, Detroit, USA). O aparelho foi calibrado antes das avaliações com o uso de um resistor de $500 \Omega$ providenciado pelo próprio fabricante. A análise da impedância bioelétrica foi realizada no período da manhã após uma noite em jejum e depois da primeira urina do dia. Os procedimentos foram realizados com o indivíduo deitado em posição supina numa superfície plana de material não condutor de eletricidade (maca) e após a retirada de calçados, meias e qualquer tipo de metal unido ao corpo (brincos, pulseiras, colares, etc.). Os eletrodos transmissores foram colocados na superfície posterior da mão direita na falange distal do terceiro metacarpo e na superfície anterior do pé direito na falange distal do segundo metatarso, e ao menos $5 \mathrm{~cm}$ de distância dos eletrodos receptores, os quais foram colocados entre o processo estilóide do rádio e da ulna, e entre os maléolos medial e lateral do tornozelo (HEYWARD e STOLARCZYK, 2000). O percentual de gordura corporal (\%GC) foi calculado por meio de equações presentes no software que acompanha - aparelho utilizado e os valores iguais ou superiores a $25 \%$ (GC $\geq 25 \%$ ) (WILLIAMS et al., 1991) foram adotados para indicar o excesso de gordura corporal.

\section{Análise Estatística}

Com o objetivo de averiguar a normalidade apresentada pelo conjunto de dados analisado, foi empregado o teste de Komolgorov-Smirnov.
Com base nas informações fornecidas por tal procedimento estatístico, observou-se o enquadramento de todas as variáveis estudadas no modelo gaussiano de distribuição, possibilitando assim a aplicação de procedimentos estatísticos para o tratamento de dados com distribuição paramétrica.

Dessa forma, a estatística descritiva utilizada no presente estudo foi composta por medidas de tendência central e dispersão, representada por valores de média e desvio padrão, respectivamente. $O$ teste qui-quadrado foi utilizado para atestar a existência de associações entre as variáveis analisadas, e a regressão logística binária, representada por valores de razão de chance $(R C)$, foi utilizada para indicar a extensão dessas associações. $O$ teste $t$ de Student para amostras independentes foi adotado para comparar valores médios entre os grupos formados. Valores de probabilidade $(P)$ inferiores a $5 \%$ foram considerados significantes estatisticamente. Todos os dados foram processados e tratados no pacote estatístico SPSS for Windows, versão 10.0 (SPSS, Inc, Chicago, IL).

\section{Resultados}

Na Tabela 1 são apresentadas informações da amostra referentes às características antropométricas, de composição corporal e ERCV de acordo com a PHAF. Foi detectado excesso de gordura corporal (GC $\geq 25 \%$ ) em $38,3 \%$ dos sujeitos, elevada quantidade de gordura subcutânea (DTR $\geq P 85$ ) em 15\%, ERCV em $48,3 \%$ e a prática insuficiente de atividades físicas (<60min/dia) em $35 \%$. Contudo, não foram verificadas diferenças entre ambos os grupos formados (ativos e inativos), nessas variáveis.

Tabela 1. Características gerais da amostra analisadas de acordo com o nível habitual de prática de atividades físicas.

\begin{tabular}{lcrcr}
\hline \multirow{2}{*}{ Variáveis } & \multicolumn{2}{c}{ Ativos $(n=39)$} & \multicolumn{2}{c}{ Inativos $(n=21)$} \\
& Média & DP & Média & DP \\
\hline Idade $($ anos $)$ & 11,7 & 1,2 & 12,1 & 1,3 \\
IMC $\left(\mathrm{kg} / \mathrm{m}^{2}\right)$ & 21,7 & 4,8 & 21,3 & 5,3 \\
CC $(\mathrm{cm})$ & 75,5 & 14,1 & 75,0 & 14,5 \\
DTR $(\mathrm{mm})$ & 12,7 & 8,3 & 13,2 & 8,2 \\
\%GC & 21,7 & 9,2 & 21,6 & 9,9 \\
\hline
\end{tabular}

$\mathrm{DP}=$ Desvio padrão; IMC= Índice de massa corporal; $\mathrm{CC}=$ Circunferência de cintura; DTR= Dobra cutânea tricipital. 
Tabela 2. Características gerais da amostra analisadas de acordo com a exposição à comportamentos sedentários.

\begin{tabular}{|c|c|c|c|c|}
\hline \multirow{2}{*}{ Variáveis } & \multicolumn{4}{|c|}{ Expostos $(n=30)$ Não Expostos $(n=30)$} \\
\hline & Média & DP & Média & DP \\
\hline Idade (anos) & 11,7 & 1,2 & 12,0 & 1,2 \\
\hline IMC (kg/m²) & 22,8 & 4,5 & 20,3 & $5,2^{*}$ \\
\hline $\mathrm{CC}(\mathrm{cm})$ & 79,4 & 12,9 & 71,2 & $14,3^{*}$ \\
\hline DTR $(\mathrm{mm})$ & 15,0 & 7,9 & 10,7 & $3,1^{*}$ \\
\hline$\% \mathrm{GC}$ & 24,4 & 9,1 & 18,9 & $9,1^{*}$ \\
\hline
\end{tabular}

${ }^{*}=\mathrm{P}<0,05 ; \mathrm{DP}=$ Desvio padrão; IMC= Índice de massa corporal; $\mathrm{CC}=$ Circunferência de cintura; DTR= Dobra cutânea tricipital.

Na Tabela 3 são apresentados os valores da regressão logística binária, onde os escores referentes à CC, DTR e \%GC são tratados como variáveis dependentes e o nível habitual de prática de atividades físicas como variável independente.

Observa-se que em todos os modelos testados, não foram observados resultados estatisticamente significativos.

Tabela 3. Regressão logística binária, com os valores de atividade física habitual como a variável independente $e$ os indicadores da composição corporal e elevado risco cardiovascular como as variáveis dependentes.

\begin{tabular}{lcccc}
\hline & & \multicolumn{3}{c}{ AFH $(\geq 60 \mathrm{~min} /$ dia $)$} \\
& & RC & $($ IC95\%) & Valor $-\mathrm{P}$ \\
\hline \%GC & $<25 \%$ & Ref. & & \\
& $\geq 25 \%$ & 0,74 & $(0,25-2,20)$ & 0,597 \\
Dtr & $<$ P85 & Ref. & & \\
& $\geq P 85$ & 2,07 & $(0,39-11,04)$ & 0,391 \\
CC & Normal & Ref. & & \\
& Elevada & 1,04 & $(0,36-3,02)$ & 0,935 \\
\hline
\end{tabular}

$\mathrm{AFH}=$ Atividade física habitual; Ref= Referência; IC95\%= Intervalo de confiança a 95\%; RC= razão de chance.

$\mathrm{Na}$ Tabela 4 são apresentados os valores da regressão logística binária, onde, os valores de CC, DTR e \%GC são tratados como variáveis dependentes e a exposição a comportamentos sedentários como variável independente. Observa-se que os jovens situados nos mais altos escores de exposição a comportamentos sedentários possuem cinco vezes mais chances de apresentar excesso de gordura corporal $(R C=$ $5,2 ; P=0,005)$ e seis vezes mais chances de apresentar ERCV $(R C=6,4 ; P=0,001)$.
Tabela 4. Regressão logística binária, com a exposição à comportamentos sedentários como a variável independente e os indicadores da composição corporal e elevado risco cardiovascular como as variáveis dependentes.

\begin{tabular}{lcccc}
\hline & \multicolumn{5}{c}{ Sedentários (expostos) } \\
& & RC & $($ IC95\%) & Valor-P \\
\hline \%GC & $<25 \%$ & Ref. & & \\
& $\geq 25 \%$ & 5,23 & $(1,65-15,51)$ & 0,005 \\
Dtr & $<$ P85 & Ref. & & \\
& $\geq P 85$ & 1,30 & $(0,31-5,40)$ & 0,718 \\
CC & Normal & Ref. & & \\
& Elevada & 6,41 & $(2,08-19,75)$ & 0,001 \\
\hline
\end{tabular}

Ref= Referência; IC95\%= Intervalo de confiança a $95 \%$; RC= razão de chance.

\section{Discussão}

No presente estudo, o percentual de jovens que apresentou o estado de ERCV $(48,3 \%)$ foi superior aos $21 \%$ observados na cidade de Presidente Prudente entre adolescentes de 11 a 17 anos (FERNANDES et al., 2007). Entretanto, esta elevada prevalência de ERCV pode ser atribuída, pelo menos em parte à forma de seleção da amostra, uma vez que os jovens procuraram 0 laboratório por acreditarem apresentar sobrepeso e obesidade.

A prática insuficiente de atividades físicas foi observada em $35 \%$ da amostra analisada, esta taxa foi inferior aos $40 \%$ observados na cidade de Pelotas - RS (HALLAL et al., 2006). No entanto, no que se refere aos dados sobre PHAF de jovens brasileiros é difícil estabelecer comparações seguras entre diferentes estudos, uma vez que tantos os métodos como os pontos de corte adotados apresentam grande variabilidade $e$, refletindo nos resultados encontrados. Nesse sentido, os pontos de corte para prática habitual de atividades físicas adotados neste estudo foram os recomendados internacionalmente (BIDDLE, CAVILL, SALLIS, 1998).

Quando são analisadas as influências da PHAF de maior intensidade nos diferentes indicadores de gordura corporal e ERCV, os resultados observados revelaram não existir associações significativas entre essas variáveis. Tais resultados estão em linha com achados anteriores, (FERNANDES et al., 2006). Entretanto, a utilização de técnicas mais precisas para avaliação da atividade física, por acelerômetro, por exemplo, Ekelund et al., (2006) verificou, entre jovens europeus, que a atividade física relacionou-se de maneira fraca com indicadores de adiposidade, mas relaciona-se 
negativamente com diferentes indicadores de risco metabólico.

No que se refere à exposição a comportamentos sedentários, o presente estudo indicou que jovens que dedicam mais tempo a atividades sedentária, apresentam chances elevadas de serem obesos e apresentar maior circunferência de cintura. Essas informações corroboram com os achados de estudos previamente publicados com adolescentes (EKELUND et al., 2006; FERNANDES et al., 2006), e reforçam a necessidade de intervenções visando à conscientização por parte das populações jovens quanto a não adoção de comportamentos sedentários.

Uma possível justificativa para esta associação entre aumento da adiposidade corporal e comportamentos sedentários, pode ser a relação entre o consumo de alimentos de alta densidade energética (LINDSAY et al., 2009), principalmente durante o período que permanecem nessas atividades sedentárias (assistir televisão, uso de computador, etc.) (GIAMMATTEl et al., 2003).

Dessa forma, o combate ao sedentarismo tem recebido crescente importância devido ao aumento no volume de estudos que têm reportado sua influência sobre a composição corporal, o desenvolvimento de diversas patologias (FERREIRA, OLIVEIRA, FRANÇA, 2007) e, também, quando se fala em prevenção de doenças crônicas e diminuição de fatores de risco, o sedentarismo constitui um fenômeno independente da prática habitual de atividades físicas, principalmente aquelas de intensidades mais elevadas (EKELUND et al., 2006; FERNANDES et al., 2008).

Nesse particular, quando se visa a elaboração de campanhas que sejam mais eficientes para o combate ao sedentarismo entre crianças e adolescentes, estudos têm indicado a importância dos hábitos dos pais sobre os hábitos dos filhos (MENDES et al., 2006). Fato que é sugerido no presente estudo e ressalta-se a necessidade de que essas campanhas alcancem também a estrutura familiar onde o jovem está inserido.

Pelo fato de Sendo assim, no que se refere ao presente estudo, indica-se que seu modelo transversal constitui sua maior limitação, uma vez que não possibilita o estabelecimento de relações de causa e efeito entre as variáveis envolvidas no estudo. Dessa forma, os autores ressaltam a escassez e a necessidade de estudos de coorte que analisem as relações entre atividades físicas de maior intensidade e comportamentos sedentários com o desenvolvimento da obesidade e fatores de risco ao desenvolvimento de DCV entre crianças e adolescentes brasileiros.

\section{Conclusão}

Com base nos resultados expostos, o presente estudo conclui que dentro do fenômeno denominado de nível habitual de prática de atividades físicas, o envolvimento com atividades físicas de maior intensidade constitui um fenômeno distinto daquele denominado de exposição à comportamentos sedentários, e que ambos influenciam de forma distinta a composição corporal. Além disso, conclui que na amostra analisada, a adoção de comportamentos sedentários apresenta maior influência na composição corporal e ERCV do que o envolvimento com atividades físicas de maior intensidade.

\section{Referências}

ALMEIDA, C.A.; PINHO, A.P.; RICCO, R.G.; ELIAS, C.P. Circunferência abdominal como indicador de parâmetros clínicos e laboratoriais ligados à obesidade infanto-juvenil: comparação entre duas referências. Jornal de Pediatria (Rio J) $2007 ; 83(2)$, p.181-5.

http://dx.doi.org/10.2223/JPED.1604

BIDDLE, S.; CAVILL, N.; SALLIS, J. Young and active? Young people and health-enhancing physical activity - evidence and implications. London: Health Education Authority; 1998.

BOUCHARD, C. The obesity epidemic: introduction. In: C. Bouchard (Ed.), Physical activity and obesity. Champaign, IL: Human Kinetics Book, 3- 20, 2000.

CHENOWETH, D.; LEUTZINGER, J. The economic cost of physical inactivity and excess weight in American adults. International Journal of Physical Activity and Health; v.3 n.2, p .14863, 2006. Disponível em:

http://www.humankinetics.com/jpah/viewarticle.cf $\underline{m}$ ?aid $=5684$

EKELUND, U.; BRAGE, S.; FROBERG, K.; HARRO, M.; ANDERSSEN, S.A.; SARDINHA, L.B.; RIDDOCH, C.; ANDERSEN, L.B. TV viewing and physical activity are independently associated with metabolic risk in children: the European Youth Heart Study. Plos Medicine 2006; v.3, 
n.12, p.2449-2457.

http://dx.doi.org/10.1371/journal.pmed.0030488

FERNANDES, R.A.; FREITAS JUNIOR, I.F.; CARDOSO, J.R.; RONQUE, E.R.V.; LOCH, M.R.; OLIVEIRA, A.R. Association between regular participation in sports and leisure time behaviors in Brazilian adolescents: A cross-sectional study. BMC Public Health 2008; 8: 329.

http://dx.doi.org/10.1186/1471-2458-8-329

FERNANDES, R.A.; OLIVEIRA, A.R.; FREITAS JÚNIOR, I.F. Correlação entre diferentes indicadores de adiposidade corporal e atividade física habitual em jovens do sexo masculino.

Revista Brasileira de Cineantropometria e Desempenho Humano V. 8 , n.4 , p.32- 8, 2006. http://www.periodicos.ufsc.br/index.php/rbcdh/arti cle/view/3929/3334

FERNANDES, R.A.; ROSA, C.S.; BUONANI, C.; OLIVEIRA, A.R.; FREITAS JÚNIOR, I.F. The use of bioelectrical impedance to detect excess visceral and subcutaneous fat. Jornal de Pediatria (Rio J) 2007; 83(6):529-34. http://dx.doi.org/10.2223/JPED.1722

FERREIRA, A.P.; OLIVEIRA, C.E.R.; FRANÇA, N.M. Síndrome metabólica em crianças obesas e fatores de risco para doenças cardiovasculares de acordo com a resistência à insulina. Jornal de Pediatria (Rio J) 2007; 83(1):21-6.

http://dx.doi.org/10.2223/JPED.1562

GIAMMATTEI, J.; BLIX, G.; MARSHAK, H.H.; WOLLITZER, A.O.; PETTITT, D.J. Television watching and soft drink consumption: associations with obesity in 11- to 13-year-old schoolchildren.

Archive of Pediatric Adolescence Medicine. 2003; 157(9):882-6. http://archpedi.amaassn.org/cgi/reprint/157/9/882

GUTIN, B.; YIN, Z.; HUMPHRIES, M.C.; BARBEAU, P. Relations of moderate and vigorous physical activity to fitness and fatness in adolescents. American Journal of Clinical Nutritrion; V.81, n.4, p.746-50, 2005.

http://www.ajcn.org/cgi/reprint/81/4/746

HALLAL, P.C.; BERTOLDI, A.D.; GONÇALVES, H.; VICTORA, C.G. Prevalence of sedentary lifestyle and associated factors in adolescents 10 to 12 years of age. Caderno de Saúde Publica 2006; 22(6):1277-87. http://dx.doi.org/10.1590/S0102$\underline{311 \times 2006000600017}$

HEYWARD, H.; STOLARCZYK, L.M. Avaliação da composição corporal aplicada. São Paulo Editora Manole 2000.

LINDSAY, A.C.; SUSSNER, K.M.; GREANEY, M.L.; PETERSON, K.E. Influence of social context on eating, physical activity, and sedentary behaviors of latina mothers and their preschoolage children. Health Educational Behavavior. 2009;36(1):81-96.

http://dx.doi.org/10.1177/1090198107308375

LOHMAN, TG, ROCHE, AF, MARTOREL, R, editors. Anthropometric standardization reference manual. Champaign: Human Kinetics Books; 1988. p. 3-8.

MENDES, M.J.; ALVES, J.G.; ALVES, A.V.; SIQUEIRA, P.P.; FREIRE, E.F. Associação de fatores de risco para doenças cardiovasculares em adolescentes e seus pais. Revista Brasileira de Saúde Materno Infantil 2006;

6(suplemento1):549-54.

http://dx.doi.org/10.1590/S1519$\underline{38292006000500007}$

MUST, A.; DALLAL, G.E.; DIETZ, W.H. Reference data for obesity: 85th and 95th percentiles of body mass index (wt/ht2) and triceps skinfold thickness. American Journal of Clinical Nutritrion 1991; 53:839-46.

http://www.ajcn.org/cgi/reprint/53/4/839?maxtosho $\mathrm{w}=\& \mathrm{HITS}=10$ \&hits $=10 \&$ RESULTFORMAT $=$ \&auth or1=must\&searchid=1\&FIRSTINDEX=0\&sortspec =relevance\&resourcetype=HWCIT

RIBEIRO, R.Q.C.; LOTUFO, P.A.; LAMOUNIER, J.A.; OLIVEIRA, R.G.; SOARES, J.F.; BOTTER, D.A. Fatores adicionais de risco cardiovascular associados ao excesso de peso em crianças e adolescentes. O estudo do coração de Belo horizonte. Arquivos Brasileiros de Cardiologia 2006; 86(6): 408- 18.

http://dx.doi.org/10.1590/S0066$\underline{782 \times 2006000600002}$

TAYLOR, R.W.; JONES, J.E.; WILLIAMS, S.M.; GOULDING, A. Evaluation of waist circumference, waist-to-hip, and the conicity index as screening tools for high trunk fat mass, as measured by dual-energy $\mathrm{X}$-ray absorptiometry, in children aged 3-19y. American Journal of Clinical Nutritrion 2000; 72:490-5. http://www.ajcn.org/cgi/reprint/72/2/490?maxtosho $\mathrm{w}=$ \&HITS=10\&hits=10\&RESULTFORMAT=\&auth or1=taylor\&andorexactfulltext=and\&searchid $=1 \& \mathrm{~F}$ IRSTINDEX=0\&sortspec=relevance\&resourcetyp $\underline{\mathrm{e}=\mathrm{HWCIT}}$

WANG, Y.; MONTEIRO, C.; POPKIN, B.M. Trends of obesity and underweight in older children and adolescents in the United States, Brazil, China, and Russia. American Journal of Clinical Nutritrion 2002; 75:971-7.

http://www.ajcn.org/cgi/reprint/75/6/971?maxtosho $\mathrm{w}=\& \mathrm{HITS}=10$ \&hits $=10 \&$ RESULTFORMAT $=$ \&auth or1=wang\&andorexactfulltext=and\&searchid $=1 \& \mathrm{~F}$ IRSTINDEX=0\&sortspec=relevance\&resourcetyp $\underline{\mathrm{e}=\mathrm{HWCIT}}$ 
WILLIAMS, D.P.; GOING, S.B.; LOHMAN, T.G.; HARSHA, D.W.; SRINIVASAN, S.R.; WEBBER, L.S.; BERENSON, G.S. Body fatness and risk for elevated blood pressure, total cholesterol, and serum lipoprotein ratios in children and adolescents. American Journal of Public Health 1992; 82(4):358-63.

http://www.ajph.org/cgi/reprint/82/3/358

Esse artigo foi apresentado em Sessão Temática no VI Congresso Internacional de Educação Física e Motricidade Humana e XII Simpósio Paulista de Educação Física, realizado pelo Departamento de Educação Física do IB/UNESP Rio Claro, SP de 30/4 a 03/5 de 2009.

\section{Endereço:}

Rômulo Araújo Fernandes

Rua Roberto Simonsen, 305.

Presidente Prudente SP Brasil

19.060-900

Telefone: (18) 3229-5345 Fax: (18) 3221-5681

e-mail: romulo_ef@yahoo.com.br

Recebido em: 10 de fevereiro de 2009.

Aceito em: 03 de abril de 2009.

\section{(c) (i) (9)}

Motriz. Revista de Educação Física. UNESP, Rio Claro, SP, Brasil - elSSN: 1980-6574 - está licenciada sob Licença Creative Commons 